\title{
Application of Improved Harris Algorithm in Sub-Pixel Feature Point Extraction
}

\author{
Zhijia Zhang, Hongliang Lu, Xin Li, Wenqiang Li, and Weiqi Yuan
}

\begin{abstract}
Harris algorithm is widely used in image feature point extraction, but the algorithm only can reach the accuracy at pixel level. Moreover, the stability of the algorithm is significantly influenced by the choice of $K$ value. In this paper, according to mathematics principles, threshold calculation method for the algorithm has been improved and the influence of $k$ selected by users on the stability of the algorithm is eliminated. On the basis of extracting feature points via Harris algorithm, a method for solving the extreme points of the intersecting line of curved surface and gradient plane has been proposed by using the fitting surface geometry model of gradation value at the near neighborhood of corner, and the positioning of feature point at sub-pixel level and application to camera calibration based on checkerboard template have been realized. It is shown with experimental results that this method is effective in improving the precision and accuracy of feature point positioning.
\end{abstract}

Index Terms-Harris algorithm, threshold, surface fitting method, contour line, extreme point, sub-pixel.

\section{INTRODUCTION AND IMPROVEMENT OF HARRIS ALGORITHM}

Currently, camera calibration method based on checkerboard template has been applied more and more widely by which the correlation between image coordinates and spatial coordinates can be determined through extracting the positional coordinates of checkerboard image corners, and thus the internal and external parameters of the camera could be obtained. The positioning accuracy of checkerboard corner directly affects the final calibration. Among the current feature point location algorithms, Harris operator based on gray value calculation is commonly used to extract feature points in image, which is simple and feasible with good corner location accuracy, translational and rotational invariance, and finds a large number of applications in camera calibration [1], [2]. However, the determination of threshold in Harris algorithm is significantly influenced by artificial selection of $\mathrm{K}$, and inappropriate threshold could result in extraction without satisfaction. This will interfere with the stability of algorithm to a certain extent, and the algorithm can only reach an extraction accuracy of pixel-level.

A novel threshold determination method has been proposed based on mathematical principles avoiding the

Manuscript received July 8, 2013; revised September 20, 2013

Z. J. Zhang, H. L. Lu, W. Q. Li, and W. Q. Yuan are with Technology Computer Vision Group, Shenyang University of Technology, Shenyang, China.

X. Li was with Liaoning Broadcasting \& TV Transmission Station, Shenyang, China. impact of artificial $k$ value selection on the stability of algorithm and verified by experimental results that this method can meet actual requirements. In order to improve positioning accuracy of Harris algorithm, corner position can be further determined by use of method of solving extreme points on surface taking advantage of the property that the model of gray value near corners is similar to surface model and ideal corner lies in the position of extreme point on surface. By solving extreme points of intersection line of surface and gradient plane, feature points were extracted at sub-pixel level in this paper, and the positioning error has been reduced to some extent. It is validated with experimental results for comparison that the precision and accuracy of this algorithm has improved to a certain extent.

There are basically two types of reverse template image corner detection algorithms. The first is to extract straight lines from template image, and then solve the point of intersection. The accuracy of this method depends significantly on the accuracy of line detection, easy to introduce errors, and it is more difficult in the case of larger camera lens distortion coefficient. The other one is corner detection method based on image gray. It is indicated by experimental results that Harris algorithm shows good robustness on the impact of image rotation, luminance and viewing angle variation, and great positioning performance, on the other hand. But the stability decreases under influence of noise.

The basic principle of Harris algorithm is: firstly, a template of $n \times n$ size as detection window is designed as required, which scans all the pixels along the grayscale image of figure. Calculate changes of gray values within the template according to equation (1), where $g x$ and $g y$ represent gradation gradient map of the image in $x$ and $y$ direction, and $G(s)$ stands for Gaussian template [3]-[7]. The matrix of Harris algorithm is given by:

$$
M=G(s) *\left[\begin{array}{cc}
g_{x}^{2} & g_{x} g_{y} \\
g_{x} g_{y} & g_{y}^{2}
\end{array}\right]
$$

where in $M$ is the autocorrelation matrix of pixels, and the two eigenvalues are $\lambda 1$ and $\lambda 2$ which are proportional to the curvature of $M$. I.e., when the two eigenvalues are relatively small, indicating that the area near target point is "flat region"; only one large eigenvalue indicating "margin region", and both large eigenvalues with significant variations in any direction indicate that target point is located in corner place. Threshold value formula of corner can be determined according to this nature, as shown in equation (2). When the variation at certain point is greater than a given threshold value, then this point can be considered as the corner to be detected. 


$$
R=\operatorname{Det}(M)-k^{*}(\operatorname{trace}(M))^{2}
$$

Det $(M)$ and trace $(M)$ in equation (2) represent determinant and trace of the matrix, and $k$ is an empirical coefficient derived from experimental $(k=0.04 \sim 0.06)$. The quality and quantity of corner extraction is dependent on the size of R-value: a too large $R$ will result in losing part of the corners; on the other hand, a too small $\mathrm{R}$ will extract some points beyond corners. Threshold value is determined by the difference in Formula (2), while the mathematical expression to determine threshold value in our work, according to the nature of the above-mentioned eigenvalues at corners is described as follows:

$$
R=\frac{4 \operatorname{Det}(M)}{1+(\operatorname{trace}(M))^{2}}
$$

where Det $(M)=\lambda 1 \times \lambda 2$, and trace $(M)=\lambda 1+\lambda 2$. When both eigenvalues are large, $R$ tends to 1 , and a larger and other smaller eigenvalue will make $R$ tend to 0 . When both eigenvalues are small and less than $1, R$ tends to 0 due to the presence of 1 in the denominator. To summarize, a point can be determined to be a corner where $R$ is close to 1 and the number of feature points could be known using the particularity of chessboard template. Feature point extraction conducted in our experiment can be described into four steps: 1) Gray gradient maps of the template in $x$ and $y$ direction were calculated. 2) Autocorrelation coefficients of all pixels were calculated according to M; 3 ) $R$ values of all the pixels were calculated according to formula (3) and the sequence was arranged following the size of $R$ values; 4 ) Corners were located in where the first $\mathrm{n}$ points of the sequence of $R$ values lie based on the set number of feature points of $n$. The effectiveness of the algorithm can be demonstrated with some experimental renderings below.

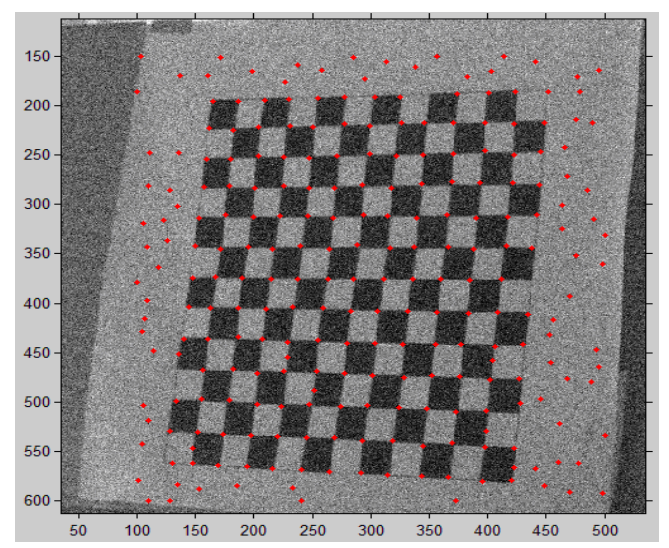

Fig. 1. Rendering of feature point extraction when $k$ equals 0.06

As can be seen from the experimental data and feature point extraction rendering, the amount of feature points extracted differs at $k=0.06$ and 0.04 using the original Harris algorithm interfered by Gaussian noise, and corner extraction has a large error under both conditions. It is clearly found from Fig. 1 and Fig. 2 that some points in the smooth region outside the board were extracted as a corner point, indicating that the original Harris algorithm is sensitive to noise. The extraction rendering by use of the proposed algorithm under the same conditions is shown in Fig. 3, from which we can see that most of the unwanted points in smooth region are removed, and corner extraction accuracy has been greatly improved. Details of the number of extracted corners in the above-described cases are exhibited in Table I.

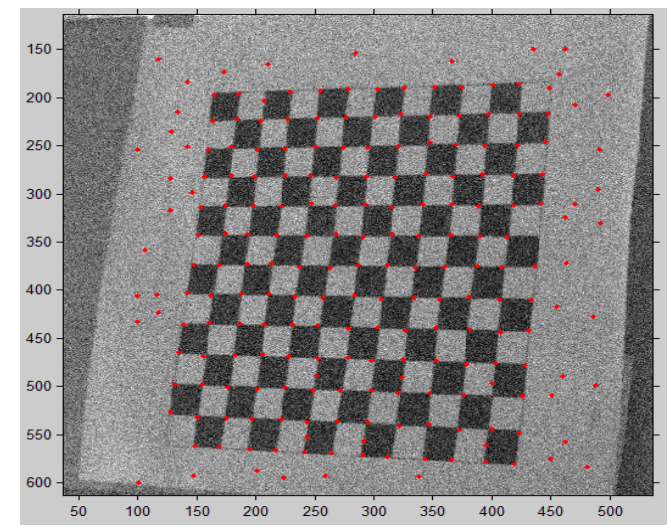

Fig. 2. Rendering of feature point extraction when k equals 0.04

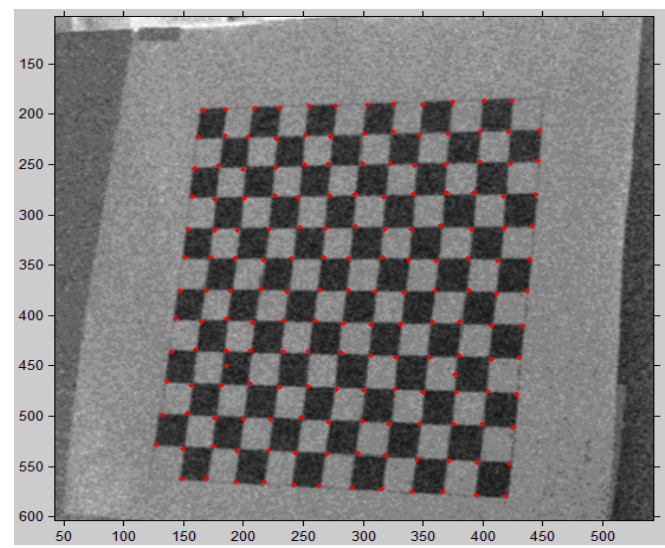

Fig. 3. Rendering of feature point extraction using the IHA-FPE algorithm

TABLE I: CONTRAST OF AMOUNTS OF EXTRACTED CORNERS

\begin{tabular}{|l|c|c|c|}
\hline Amount & $\begin{array}{c}\text { Original Harris } \\
\text { algorithm } \\
(k=0.06)\end{array}$ & $\begin{array}{c}\text { Original Harris } \\
\text { algorithm } \\
(k=0.04)\end{array}$ & $\begin{array}{c}\text { Algorithm } \\
\text { proposed in } \\
\text { this paper }\end{array}$ \\
\hline Actual corners & 169 & 169 & 169 \\
\hline Extracted corners & 251 & 212 & 168 \\
\hline
\end{tabular}

\section{FITTING AND EXTREME POINT EXTRACTION}

Fitting the edge point is a method to fit gray value at the edge of image according to a certain edge model ensuring the smallest error and thereby obtain an edge of sub-pixel accuracy [8]-[10]. A specified analytical formula is used in fitting method to reconstruct continuous image gray distribution. Usually surface fitting method is commonly utilized in feature point extraction, which applies a binary quadratic polynomial to simulating the feature point function $C(x, y)$ :

$$
C\left(x_{i}, y_{j}\right)=a_{0}+a_{1} x_{i}+a_{2} y_{j}+a_{3} x_{i}^{2}+a_{4} x_{i} y_{j}+a_{5} y_{j}^{2}
$$

Sub-pixel level extraction via surface fitting method can be divided into two steps: a set of equations including $\mathrm{n} \times \mathrm{n}$ equations can be generated from an $n \times n$ fitting window, and finally undetermined coefficients $a_{0}, a_{1}, a_{2}, a_{3}, a_{4}$ and $a_{5}$ of conicoid will be solved with the least squares method. For example, a $3 \times 3$ fitting window can be written in matrix form as follows: 


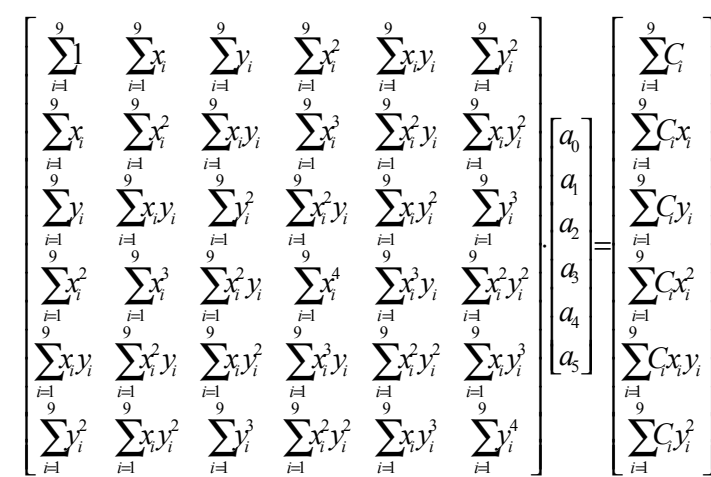

Extreme points of quadratic polynomial function $C(x, y)$ on fitting surface satisfy the following equations:

$$
\left\{\begin{array}{l}
\frac{\partial c(x, y)}{\partial x}=a_{1}+2 a_{3} x+a_{4} y=0 \\
\frac{\partial c(x, y)}{\partial y}=a_{2}+2 a_{5} x+a_{4} y=0
\end{array}\right.
$$

Thus, positions of the extreme points of fitting surface can be obtained according to the following equations:

$$
\left\{\begin{array}{l}
x=\frac{2 a_{1} a_{5}-a_{2} a_{5}}{a_{4}^{2}-4 a_{3} a_{5}} \\
y=\frac{2 a_{1} a_{3}-a_{1} a_{4}}{a_{4}^{2}-4 a_{3} a_{5}}
\end{array}\right.
$$

It is demonstrated through experiments that there is difference between position of feature points obtained by calculation and the actual one. This is because extreme points of the surface model obtained may be not global extreme points but just local ones, while the actual feature point lies in the location of global extreme point on fitting surface, so error occurs. In this paper, a plane containing extreme points of surfaces can be determined by the combination of gradient vector through equation (8) and tangent vector of the contour at obtained extreme points. An accurate position of feature points can be further derived by solving extreme points of the intersection line of this plane and surface.

$$
\operatorname{grad} f=\left\{\frac{\partial c(x, y)}{\partial x}, \frac{\partial c(x, y)}{\partial y}\right\}=\frac{\partial c(x, y)}{\partial x} i+\frac{\partial c(x, y)}{\partial y} j
$$

\section{Sub-PiXel Feature PoInt EXTRACtion Using Harris ALGORITHM}

Generally, the accuracy of Harris algorithm to extract feature points only reaches pixel-level, this article will be legitimate positioning extreme point method is applied to the Harris algorithm to achieve the extraction of sub-pixel accuracy. An improved Harris algorithm combined with fitting-positioned-extreme point metho (IHA-FPE, for short) was employed in our experiment of this paper to achieve the extraction of sub-pixel feature points in image. The specific procedures are given below:

1) Coordinates of the initial feature points were obtained by the use of improved Harris algorithm.

2) A $3 \times 3$ matrix was configured and centered on the coordinates of initial feature point, and took any 6 groups of coordinates into equation (5) to obtain $a_{0}, a_{1}, a_{2}, a_{3}, a_{4}$ and $a_{5}$ for formula (6) and (7). Then the location of initial sub-pixel feature points was calculated.

3) Solved gradient vector and contour tangent vector at the coordinates of initial sub-pixel-level feature points.

4) The intersection line of the plane determined by the combination of gradient vector and tangent vector of the contour and surface was obtained.

5) The extreme point of the intersection line was considered as the coordinate position of sub-pixel feature point.

As shown in Fig. 4, OA is the gradient vector at extreme point and $\mathrm{OC}$ tangent vector of contour at extreme point, $\mathrm{OB}$ normal vector of plane $\mathrm{AOC}$, so $\mathrm{AOB}$ is the plane required. For comparison, $\mathrm{B}$ is the position of initial extreme point while $\mathrm{A}$ is the ideal position of feature point (Fig. 5). A perspective drawing of feature point extraction experiment is shown in Fig. 6, and Fig. 7 is the enlargement of feature point extraction of No. 94.

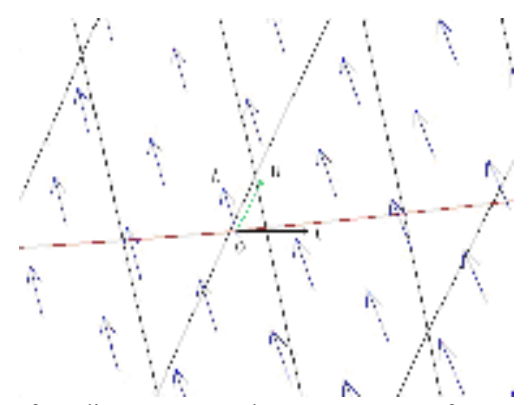

Fig. 4. Diagram of gradient vector and tangent vector of contour at extreme point

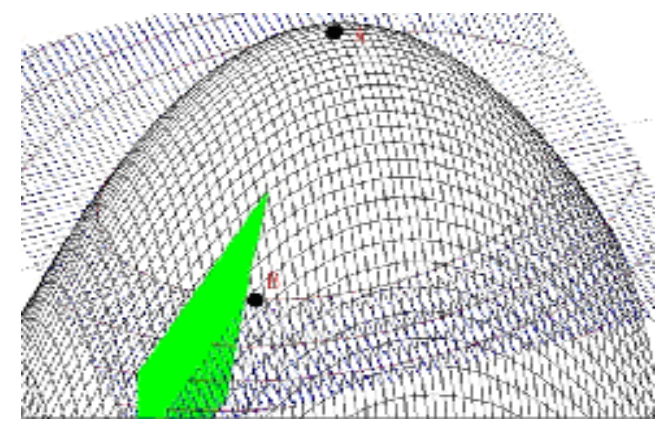

Fig. 5. Schematic diagram of the intersection between gradient plane and surface

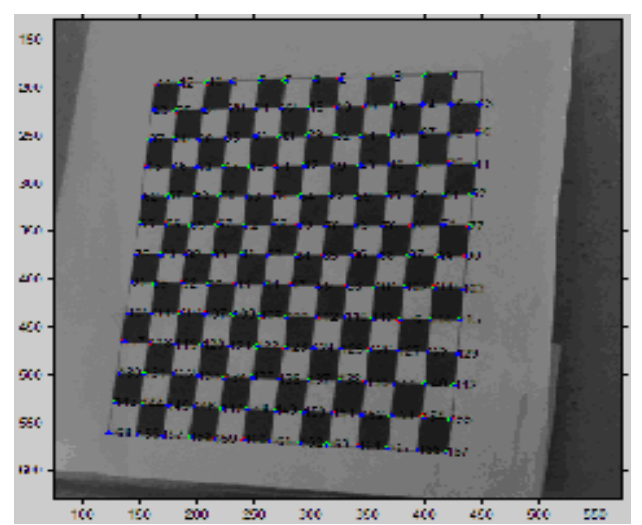

Fig. 6. Experimental rendering of feature point extraction

Experimental results are shown in Fig. 7. The red point represents the feature point positioned by Harris algorithm, the green one the positioned extreme point at the first time, and the blue one the location of extreme point positioned via IHA-FPE method. It can be clearly observed that the blue point is the closest one to the location of ideal corner. 
Coordinates of certain feature points extracted using the three different algorithms are listed in Table II.

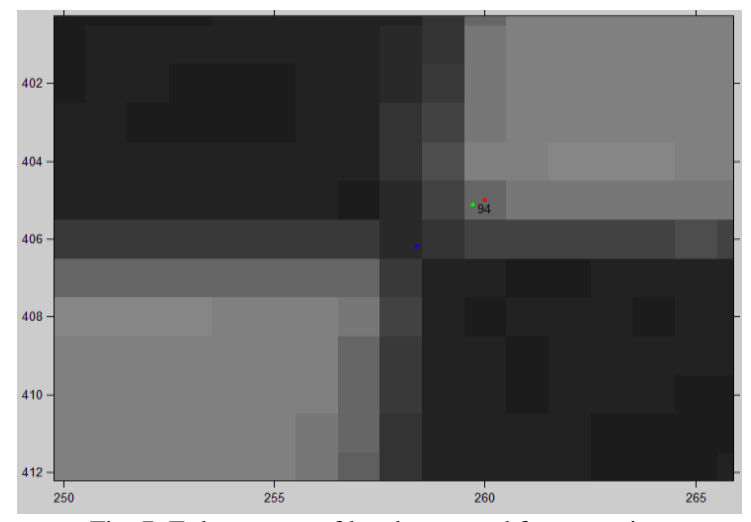

Fig. 7. Enlargement of local extracted feature points

TABLE II: CONTRAST OF COORDINATES OF EXTRACTED FEATURE POINTS USING THREE ALGORITHMS

\begin{tabular}{|c|c|c|c|c|c|c|}
\hline \multirow{2}{*}{$\begin{array}{c}\text { Algorith } \\
\text { No }\end{array}$} & \multicolumn{2}{|c}{$\begin{array}{c}\text { Harris } \\
\text { algorithm }\end{array}$} & \multicolumn{2}{c|}{ Surface fitting } & \multicolumn{2}{c|}{ IHA-FPE } \\
\cline { 2 - 7 } & $x 1$ & $y 1$ & $x 2$ & $y 2$ & $x 3$ & $y 3$ \\
\hline 1 & 424 & 186 & 424.2782 & 186.0311 & 424.4364 & 186.9251 \\
\hline 14 & 397 & 217 & 397.4733 & 217.1684 & 397.5216 & 217.9983 \\
\hline 30 & 370 & 249 & 370.3661 & 249.204 & 370.4697 & 250.0368 \\
\hline 43 & 344 & 281 & 343.4729 & 280.8446 & 343.5106 & 281.2681 \\
\hline 55 & 316 & 312 & 316.3940 & 312.3108 & 316.6432 & 312.5686 \\
\hline 70 & 289 & 343 & 289.3601 & 343.8554 & 289.9821 & 344.3226 \\
\hline 83 & 262 & 375 & 262.1004 & 374.8158 & 262.2461 & 374.6582 \\
\hline 94 & 260 & 405 & 259.7149 & 405.7444 & 258.4684 & 406.1827 \\
\hline 108 & 232 & 437 & 231.8745 & 436.7071 & 232.7688 & 435.8725 \\
\hline 120 & 204 & 469 & 204.5558 & 468.6929 & 204.3528 & 468.5489 \\
\hline 133 & 202 & 501 & 201.5530 & 500.9737 & 200.6439 & 500.8761 \\
\hline 144 & 152 & 530 & 151.5482 & 530.1277 & 149.8997 & 531.0364 \\
\hline
\end{tabular}

\section{CONCLUSIONS}

The instability caused by $\mathrm{k}$ value and sensitivity to noise of Harris algorithm for the extraction of feature points are eliminated by improving the determination form of threshold in algorithm- substituting the original mathematical form of difference for that of ratio can avoid the sensitivity of k value selected artificially to noise to some extent. Sub-pixel feature point extraction was performed by solving extreme point of the intersection line of gradient plane and surface at original feature point. In this paper, the effectiveness of the application of this method to camera calibration based on checkerboard template is verified through experimental simulation. It is noted that an additional step of fitting for extreme points in IHA-FPE algorithm will have impact on feature point extraction time.

\section{ACKNOWLEDGMENT}

Thank editors and anonymous reviewers for their careful and constructive suggestions to this paper. This work was financially supported by Shenyang Science and Technology Foundation (Grant No.F13-316-1-38, 'Research on affine invariant shape recognition based on sub-space'), Liaoning Provincial Natural Science Foundation of China (Grant No.20102163) and National Natural Science Foundation of China (Grant No. 61271365).

\section{REFERENCES}

[1] Z. L. Cheng, L. B. Gu, H. Deng, "A new method of chessboard grid corner detection," Journal of China's Manufacturing Industry Informatization, vol.39, no. 9, pp, 74-77, 2010.

[2] Y. Zhang, K. L. Fang, Y. X. Liu, "Harris algorithm-based corner detection on black white checkerboard," Computer Applications and Software, vol. 27, no. 8, pp. 251-254, 2010.

[3] Z. S. Feng and M. Q. Wang, "An image matching algorithm base on Harris and improved SIFT algorithm," Journal of Fuzhou University (Natural Science Edition), vol.40, no. 2, pp. 176-180, 2012.

[4] X. F. Xu and J. Q. Tan, "An improved multi-scale Harris feature point detection method," Computer Engineering, vol. 38, no. 17, pp. 19-21, 2012.

[5] N. Liu, R. S. Lu, and R. X. Xia, "A novel self-correcting algorithm for false corner of harris operator on calibration image," ACTA Photonica Sinica, vol. 41, no. 10, pp. 1222-1228, 2012.

[6] Y. Liu and Z. R. Zou, "The comparison of several feature point detection methods in photographic survey," Geomatics and Spatial Information Technology, vol. 19, no. 5, pp. 130-133, 2009.

[7] Y. M. Mao, M. H. Lan, and Y. Q. Wang, "An improved corner detection method based on Harris," Computer Technology and Development, vol. 19, no. 5, pp. 130-133, 2009.

[8] C. Harris and M. Stephens, "A combined corner and edge detector," in Proc. of Fourth Alvey Vision Conference, 1998, PP. 147-151.

[9] Paul Bao, Lei Zhang, and Xiaolin Wu, "Canny edge detection enhancement by scale multiplication," IEEE Trans. On Pattern and Machine Intelligence, vol. 27, no. 8, pp. 1485-1490, 2005.

[10] Y. H. He and G. C. Cai, "A new method of corner detection," Journal of Yunnan Nationalities University, Natural Sciences Edition.

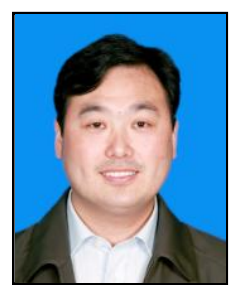

Zhijia Zhang received the B.E. and M.E. degrees from Northeastern University, Shenyang, China in 1996 and 2002 respectively. In 2006, he graduated from Shenyang Institute of automation, which attached to the Chinese academy of sciences, and received his $\mathrm{Ph} . \mathrm{D}$. degree in pattern recognition and intelligent system profession. Currently, he is a vice president working in Shenyang University of technology. His present research is focused on image processing and target recognition. 